\title{
Organisatorische und technische Entwicklungen in der Nährstoffbewirtschaftung
}

\begin{abstract}
Nährstoffe, wie Stickstoff und Phosphor, sind Wert- und Schadstoffe - diese Janusköpfigkeit wurde häufig erwähnt. Beide Stoffe sind für Lebewesen essenziell und können nicht durch andere Nährstoffe ersetzt werden. Beide Stoffe verbindet, dass sie derzeit durch die Volkswirtschaft eher linear verwendet werden, d. h., dass mit Ausnahme der innerbetrieblichen Güllenutzung in der Landwirtschaft keine ausgeprägten Kreisläufe für sie existieren. Der wesentliche Unterschied ist, dass die langfristige Verfügbarkeit von Stickstoff gegeben ist, während die natürlichen Phosphorlagerstätten geopolitisch ungünstig verteilt sind und ihre Quantität und Qualität stetig abnimmt. Damit ist die langfristige Versorgung mit ausreichend Phosphor weniger gesichert. Die Aufgabe einer optimierten Nährstoffbewirtschaftung besteht darin, durch ein Bündel an organisatorischen und technischen Maßnahmen den Nährstoffhaushalt einer Volkswirtschaft so zu gestalten, dass die Ziele „Schutz von Mensch und Umwelt“ und „effiziente Nutzung von Ressourcen“ unter Einhaltung ökonomischer und sozio-kultureller Erfordernisse erreicht werden können. In diesem Heft der Österreichischen Wasser- und Abfallwirtschaft werden ausgewählte Themen und Problemstellungen im Bereich der Nährstoffbewirtschaftung behandelt.
\end{abstract}

Schrittwieser und Kollegen gehen darauf ein, dass eine vordringliche Bewirtschaftungsfrage im Donauraum die Reduktion der Nährstoffeinträge zur Erreichung des guten Zustands der Donau und des Schwarzen Meeres ist. Sie beschreiben die Grundlagen und Zielsetzungen des Nährstoffmanagements, stellen den derzeitige Zustand der Nährstoffbelastung und die Eintragspfade dar und geben einen Überblick über die Maßnahmen zur Reduktion der Nährstoffeinträge. In den DonauUnterliegerstaaten werden derzeit große Investitionen in Abwasserreinigungsanlagen getätigt. Ein wesentlicher Erfolg ist die EU-weite Begrenzung des Phosphatgehaltes von Haushaltswaschmitteln. Über die Europäische Umweltpolitik und die gemeinsame Agrarpolitik soll nun eine gewässerschonende Landwirtschaft forciert werden. Bewusstseinsbildung und ein sogenanntes „Greening“ der gemeinsamen Agrarpolitik sind im Gange.

Egle und Kollegen vergleichen Verfahren zur Rückgewinnung von Phosphor aus Abwasser und Klärschlamm anhand naturwissenschaftlich-technischer und ökonomischer Kriterien. Ihre Arbeit zeigt, dass Technologien zur P-Rückgewinnung aus dem Schlammwasser bereits heute einsetzbar sind und unter gewissen Voraussetzungen wirtschaftlich betrieben werden können. Allerdings ist das realisierbare volkswirtschaftliche Phosphor-Rückgewinnungspotenzial vergleichsweise gering. Verfahren, die beim Klärschlamm ansetzen, weisen entweder hohe Verbräuche an Betriebsmitteln und daraus resultierende hohe Gesamtkosten auf oder sind technisch problematisch. Mit dem Ziel einer größtmöglichen Nutzung des abwasserbürtigen Phosphors wäre zukünftig eine Rückgewinnung aus Klärschlammaschen anzustreben, wobei einzelne Technologien bereits in der Nähe einer betriebswirtschaftlich sinnvollen Umsetzung sind.

Lederer und Kollegen untersuchen anhand von Stoffflussanalysen die Nährstoffsituation in einer ostugandischen Region. Die dortige Situation ist charakterisiert durch einen stetigen Anstieg der Bevölkerung bei gleichzeitiger negativer Nährstoffbilanz der Ackerböden. Ihre Ergebnisse zeigen, dass eine Reduktion der Erosion und Auswaschung, Fruchtwechsel und Feldrotation sowie bessere Verwendung von Wirtschaftsdüngern das größte Ausgleichspotenzial haben. Entgegen den Annahmen der lokalen Entscheidungsträger haben Siedlungs- und Markabfälle aus urbanen Gebieten demgegenüber ein weitaus geringeres Potenzial zum Ausgleich des Ackerbodennährstoffdefizits.

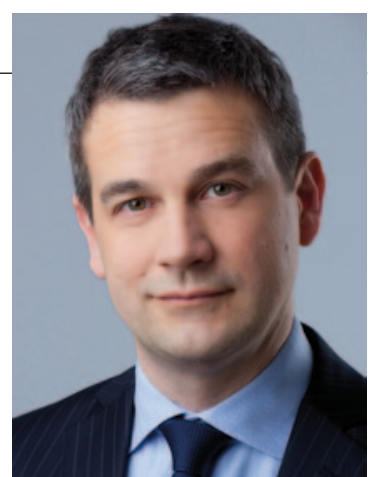

Univ.-Prof. DI Dr. Helmut Rechberger

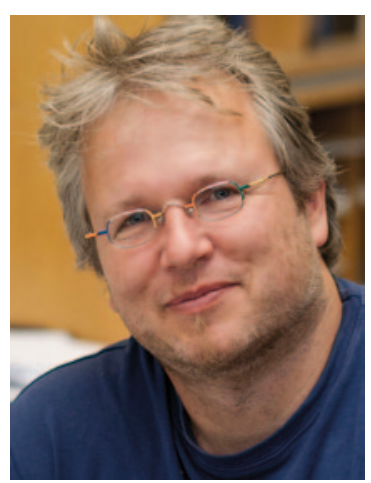

A.o.Univ.-Prof. DI Dr. Matthias ZessnerSpitzenberg 


\section{editorial}

Aus der Studie ergeben sich demnach klare Handlungsanweisungen in Richtung Optimierung der landwirtschaftlichen Praxis.

Zessner und Kollegen zeigen, dass eine umfassende Kenntnis des Zusammenhanges zwischen menschlicher Aktivität, den daraus resultierenden Emissionen und den Auswirkungen auf die Gewässer eine wesentliche Grundlage ist, um die Effektivität von Maßnahmen zur Verringerung der Phosphorbelastung von Gewässern auf Ebene von Einzugsgebieten abschätzen zu können. Darauf aufbauend können lokale Maßnahmenwirkungen zur Emissionsreduktion bewertet, das Potenzial für eine Umsetzung der Maßnahmen in einer Region aufgrund der vorherrschenden Gebietskulisse und Landbewirtschaftung erhoben und die Maßnahmenwirksamkeiten im Kontext von Gesamtemissionen und Retention dargestellt werden. Ergebnisse entsprechender Untersuchungen in Oberösterreich zeigen unter anderem, dass Erosionsschutzmaßnahmen bzw. Maßnahmen zur Vermeidung von erosiven Phosphoreinträgen in Fließgewässer vor allem dann ein hohes Potenzial zur Reduktion von Phosphorbelastungen von Gewässern haben, wenn sie gezielt verortet werden.

Wir freuen uns, dass es möglich war dieses Heft zusammenzustellen, möchten uns bei allen bedanken, die dazu beigetragen haben und hoffen, dass die Artikel bei den Leserinnen und Lesern der ÖWAW auf Interesse stoßen.

\section{Univ.-Prof. DI Dr. Helmut Rechberger}

A.o.Univ.-Prof. DI Dr. Matthias Zessner-Spitzenberg

Technische Universität Wien

Institut für Wassergüte, Ressourcenmanagement und Abfallwirtschaft

Karlsplatz 13/226

1040 Wien

helmut.rechberger@tuwien.ac.at

mzessner@iwag.tuwien.ac.at 